\title{
A Síndrome de Down ante a infecção por Coronavírus
}

\section{The Down Syndrome in the face of Coronavirus infection \\ La Síndrome de Down frente a la infección por Coronavirus}

Larissa Távore Silva ORCID: https://orcid.org/0000-0002-5837-7324 União das Faculdades dos Grandes Lagos, Brasil E-mail: larissatavoree@gmail.com

João Pedro Granado Leme Nunes ORCID: https://orcid.org/0000-0003-3431-1870 União das Faculdades dos Grandes Lagos, Brasil E-mail: jp_granado@hotmail.com

Alex Gomes de França ORCID: https://orcid.org/0000-0003-1808-1294 União das Faculdades dos Grandes Lagos, Brasil E-mail: alex_gomes182@hotmail.com

Alexandre Silva Zacarias

ORCID: https://orcid.org/0000-0001-9369-3405 União das Faculdades dos Grandes Lagos, Brasil E-mail: alexandreszacarias@gmail.com Yasmin Abou Rahal

ORCID: https://orcid.org/0000-0001-6726-3989 União das Faculdades dos Grandes Lagos, Brasil E-mail: yasmin.rahal @ hotmail.com

Gabriela Andrade Roversi ORCID: https://orcid.org/0000-0002-0214-2198 União das Faculdades dos Grandes Lagos, Brasil E-mail: gabi_roversi@hotmail.com

Beatriz Barboza Garavaso ORCID: https://orcid.org/0000-0002-6506-4713 União das Faculdades dos Grandes Lagos, Brasil

E-mail: beatriz_garavaso@ @otmail.com

Larissa Barros de Almeida ORCID: https://orcid.org/0000-0003-2364-9892 União das Faculdades dos Grandes Lagos, Brasil

E-mail: larissabarrosfai@gmail.com

Maria Júlia Escame Romano ORCID: https://orcid.org/0000-0003-0078-2666 Universidade Brasil, Brasil

E-mail: mariajulia.romano@ hotmail.com Adriani Monisi Visoná

ORCID: https://orcid.org/0000-0003-2735-0070 Universidade Brasil, Brasil

E-mail: moni25_1998@hotmail.com Julia Coradini Virgili

ORCID: https://orcid.org/0000-0001-6667-0400 Universidade Brasil, Brasil E-mail: coradini.ju@gmail.com

Isabella Cavalari Barboza ORCID: https://orcid.org/0000-0001-8964-861X Universidade Brasil, Brasil

E-mail: isabella.cavalari@hotmail.com

Yanca Lucrécia Moreira Schmidt ORCID: https://orcid.org/0000-0001-8604-0502 Universidade Brasil, Brasil

E-mail: yancamoreira19@hotmail.com

Fábio Gonçalves

ORCID: https://orcid.org/0000-0002-5474-852X

Universidade do Oeste Paulista, Brasil

E-mail: fabiogoncalves19892012@gmail.com

Elenberg Chaves de Paula

ORCID: https://orcid.org/0000-0003-4170-7726

União das Faculdades dos Grandes Lagos, Brasil

E-mail: elenbergde@hotmail.com 


\begin{abstract}
Resumo
Este estudo teve como objetivo elucidar de que forma as alterações genéticas provocadas pela Síndrome de Down (SD) impactam nos casos de acometimento pelo SARS-CoV-2, além de expor como o isolamento social pode afetar negativamente a vida de indivíduos com SD. O estudo adotou o modelo de revisão literária qualitativa, através das bases de dados Google Acadêmico, SciELO e PubMed, por meio dos descritores "Down Syndrome" e "Coronavirus", sendo selecionados vinte artigos. Percebeu-se que a SD é a forma genética mais comum de deficiência intelectual e as alterações que a trissomia do cromossomo 21 acarretam, impactam significativamente no curso da doença de pessoas com SD infectadas pelo Coronavírus, já que o sistema imune delas é hiperativado, com capacidade para gerar uma chuva de citocinas, que facilitará a entrada do vírus nas células, favorecendo, assim, a replicação viral. Ademais, notou-se que, mesmo o isolamento social sendo eficaz no combate à pandemia, ele pode acarretar diversos danos à saúde da população com SD, o que pode agravar certas comorbidades pré-existentes nesse grupo. Portanto, é muito relevante a vigilância e a capacitação dessa população e de suas famílias, para que previnam-se de forma correta com técnica eficaz de lavagem de mãos e uso de equipamentos de barreira - minimizando a exposição ao vírus; e a socialização e a prática de atividade física - mesmo durante os períodos de quarentena e de forma remota - de maneira que preserve-se a saúde emocional e física desses indivíduos.
\end{abstract}

Palavras-chave: Coronavírus; Síndrome de Down; COVID-19.

\begin{abstract}
This study aimed to elucidate how the genetic alterations caused by Down Syndrome (DS) impact cases of involvement by SARS-CoV-2, in addition to showing how social isolation can negatively affect the lives of individuals with DS. The study adopted the qualitative literary review model, through the Google Academic, SciELO and PubMed databases, using the descriptors "Down Syndrome" and "Coronavirus", with twenty articles being selected. It was noticed that DS is the most common genetic form of intellectual disability and the changes that trisomy 21 cause significantly impact the disease course of people with DS infected with Coronavirus, since their immune system is overactivated, with ability to generate a shower of cytokines, which will facilitate the entry of the virus into cells, thus favoring viral replication. Furthermore, it was noted that, even though social isolation is effective in combating the pandemic, it can cause several damages to the health of the population with DS, which can aggravate certain pre-existing comorbidities in this group. Therefore, the surveillance and training of this population and their families is very important, so that they can correctly prevent themselves - with an effective hand washing technique and use of barrier equipment - minimizing exposure to the virus; and socialization and physical activity - even during quarantine periods and remotely - in order to preserve the emotional and physical health of these individuals
\end{abstract}

Keywords: Coronavirus; Down syndrome; COVID-19.

\title{
Resumen
}

Este estudio tuvo como objetivo dilucidar cómo las alteraciones genéticas provocadas por el Síndrome de Down (SD) impactan en los casos de afectación por SARS-CoV-2, además de mostrar cómo el aislamiento social puede afectar negativamente la vida de las personas con SD. El estudio adoptó el modelo de revisión literaria cualitativa, a través de las bases de datos Google Academic, SciELO y PubMed, a través de los descriptores "Síndrome de Down" y "Coronavirus", seleccionándose veinte artículos. Se notó que el SD es la forma genética más común de discapacidad intelectual y las alteraciones que ocasiona la trisomía 21 impactan significativamente en el curso de la enfermedad de las personas con SD infectadas por Coronavirus, ya que su sistema inmunológico está sobreactivado, con capacidad para generar una lluvia de citocinas. lo que facilitará la entrada del virus en las células, favoreciendo así la replicación viral. Además, se observó que, si bien el aislamiento social es efectivo para combatir la pandemia, puede ocasionar varios daños a la salud de la población con SD, lo que puede agravar ciertas comorbilidades preexistentes en este grupo. Por lo tanto, la vigilancia y capacitación de esta población y sus familias es muy importante, para que puedan prevenirse correctamente - con una técnica eficaz de lavado de manos y uso de equipos de barrera - minimizando la exposición al virus; y socialización y actividad física, incluso durante los períodos de cuarentena y de forma remota, para preservar la salud emocional y física de estas personas.

Palabras clave: Coronavirus; Sindrome de Down; COVID-19.

\section{Introdução}

Em 11 de março de 2020, a Organização Mundial da Saúde (OMS) declarou o novo Coronavírus como uma pandemia. A doença é transmitida, predominantemente, por gotículas ou fômites, e pode cursar com comprometimento pulmonar leve a grave, com sintomas que podem incluir tosse, febre, coriza, dor de garganta e dispneia. Apesar da letalidade da doença ser mais baixa se comparada a outros Coronavírus, sua alta transmissibilidade tem ocasionado um maior número absoluto de mortes do que a combinação das epidemias produzidas pelos SARS-CoV e o MERS-CoV (Aquino et al., 2020;

Silva et al., 2021). 
Estudos recentes demonstraram que infecções de Síndrome Respiratória Aguda Grave (SARS) por Coronavírus II, são acarretadas por resposta imune exacerbada e descontrolada do vírus, ativando a cascata de citocinas, que gera síndrome do desconforto respiratório agudo, processos tromboembólicos e falência de múltiplos órgãos (Kantar et al., 2020). Embora 80\% dos casos evoluam de forma leve, a forma severa demonstrou acometer mais frequentemente pessoas idosas e/ou com comorbidades - dentre elas, a Síndrome de Down (SD). Assim, este trabalho tem como objetivo apresentar as principais alterações genéticas que acometem o sistema imune de pessoas com SD e, portanto, fazem com que essa população seja alvo de uma evolução mais grave ante um contágio pelo Coronavírus.

A SD é a forma genética mais comum de deficiência intelectual e indivíduos com este tipo de trissomia apresentam comorbidades, tais como obesidade, diabetes tipo I e doença cardíaca congênita, que estão associadas a um pior prognóstico da COVID-19 (De Toma; Dierssen, 2021). A prevalência de doenças cardiovasculares nessas pessoas é de 40 a $50 \%$ e existe também maior propensão ao sobrepeso e à obesidade; bem como presença de alterações nas vias aéreas, o que facilita a infecção pelo vírus. Ademais, ocorre variação do fenótipo imunológico com a idade, em razão de alteraçães na regulação de citocinas; assim, as crianças são mais suscetíveis, enquanto os adultos frequentemente apresentam aumento dos biomarcadores pró-inflamatórios. Essas alterações podem ter impacto nas doenças anatômicas dos pacientes e aumentar a prevalência de condições inflamatórias crônicas, cardiopatias, doenças autoimunes e mortalidade por sepse (Faundes et al., 2021; Russo et al., 2020; Vita et al., 2020), de modo que as alterações anatômicas propiciam a colonização e, com isso, a infecção de vias aéreas superiores, bem como as cardiopatias influenciam no desfecho grave da COVID-19, acarretando uma maior mortalidade. No entanto, especula-se que infecções virais repetidas nos primeiros anos de vida podem aumentar a imunidade humoral e celular natural, o que explicaria a diminuição das infecções com a idade (Correia et al., 2020; Krishnan et al., 2020; Kim-Hellmuth et al., 2021).

Em pacientes com trissomia do cromossomo 21, a entrada e a multiplicação do SARS-CoV-2 podem ser facilitadas, devido à triplicação de TMPRSS2, que produz aumento da ativação da proteína $S$ viral e regulação negativa das junções fechadas, já que elas dificultam a endocitose viral (Kantar et al., 2020). Além disso, observou-se que as células T CD8+, em pessoas com SD, superproduzem IL-10 e células pró-inflamatórias, como TNF- $\alpha$, IFN- $\gamma$, MIP-1 ${ }^{\mathrm{a}}$, IL-2, IL-8, MIP-1b e eotaxina (Espinosa, 2020).

Atualmente, para prevenir a infecção pelo Coronavírus é necessário que evite-se a exposição ao vírus: os achados científicos sugerem fortemente que o isolamento de casos, combinados à quarentena e ao distanciamento social, têm o potencial de diminuir a transmissão da doença. Todavia, indivíduos com SD necessitam de socialização para uma melhor qualidade de vida e autonomia, de maneira que o isolamento social pode representar um grande desafio para essa população (Aquino; Lima, 2020; Russo et al., 2020).

\section{Metodologia}

Este trabalho é uma revisão bibliográfica qualitativa, já que as informações apresentadas foram pautadas em levantamentos bibliográficos, nos quais o próprio pesquisador é o instrumento principal durante todo o processo, o que facilita a compreensão dos estudos (Soares et al., 2018).

A análise bibliográfica foi realizada por meio das bases de dados Google Scholar, PubMed e SciELO. Os descritores utilizados na busca foram, respectivamente, "Impacto da COVID-19 em pacientes com Síndrome de Down" e "Down Syndrome" e "Coronavirus", por meio do operador booleano AND. Incluíram-se artigos completos e gratuitos publicados até o atual ano (2021), em todos os idiomas que abordassem o tema preterido. 


\section{Resultados e Discussão}

Nas bases de dados Google Scholar, PubMed e SciELO foram encontrados, respectivamente: 91.200 artigos, 14 artigos e 15 artigos que, a partir da análise de título e, de acordo com a proximidade ao tema proposto, foram selecionados 20 artigos, os quais foram utilizados nesta revisão.

Posteriormente, com intuito de facilitar a análise dos dados obtidos, os artigos selecionados foram organizados, por ordem alfabética, em tabela (Quadro 1) com os seguintes elementos: autor, título, objetivo e perfil da amostra.

Quadro 1 - Artigos e elementos principais.

\begin{tabular}{|c|c|c|c|}
\hline AUTOR & TÍTULO & OBJETIVO & PERFIL DA AMOSTRA \\
\hline Aquino; Lima, 2020 & $\begin{array}{l}\text { Medidas do distanciamento } \\
\text { social no controle da pandemia } \\
\text { de COVID-19: Potenciais } \\
\text { impactos e desafios no Brasil }\end{array}$ & $\begin{array}{l}\text { Sistematizar as evidências sobre } \\
\text { o impacto das medidas de } \\
\text { distanciamento social na } \\
\text { epidemia de COVID-19 e } \\
\text { discutir sua implementação no } \\
\text { Brasil. }\end{array}$ & Revisão narrativa \\
\hline Clift et al., 2021 & $\begin{array}{l}\text { COVID-19 Mortality Risk in } \\
\text { Down Syndrome: Results From } \\
\text { a Cohort Study of } 8 \text { Million } \\
\text { Adults }\end{array}$ & $\begin{array}{l}\text { Avaliar a Síndrome de Down } \\
\text { como um fator de risco para } \\
\text { morte por COVID-19, por meio } \\
\text { de uma análise abrangente de } \\
\text { dados em nível individual em } \\
\text { um estudo de coorte de } 8,26 \\
\text { milhões de adultos (com idade> } \\
19 \text { anos). }\end{array}$ & Estudo de coorte \\
\hline Correia et al., 2020 & $\begin{array}{llr}\text { Protagonismo de } & \text { jovens } & \text { com } \\
\text { Síndrome de } & \text { Down } & \text { em } \\
\text { campanha de prevenção } & \text { ao } \\
\text { SARS-CoV-2 } & & \end{array}$ & $\begin{array}{l}\text { Visibilidade à população Down } \\
\text { promovendo sua capacidade em } \\
\text { conscientização social. }\end{array}$ & $\begin{array}{l}\text { Campanha com abordagem } \\
\text { qualitativa }\end{array}$ \\
\hline De Cauwer; Spaepen, 2021 & $\begin{array}{l}\text { Are patients with Down } \\
\text { Syndrome vulnerable to life- } \\
\text { threatening COVID-19? }\end{array}$ & $\begin{array}{l}\text { Descrever o curso clínico de } 4 \\
\text { pacientes com Síndrome de } \\
\text { Down durante o surto de } \\
\text { COVID-19. }\end{array}$ & Relato de caso \\
\hline De Toma; Dierssen, 2021 & $\begin{array}{l}\text { Network analysis of Down } \\
\text { Syndrome and SARS-Cov-2 } \\
\text { identifies risk and protective } \\
\text { factors for COVID-19 }\end{array}$ & $\begin{array}{l}\text { Obter informações sobre a } \\
\text { interação entre os genes da } \\
\text { Síndrome de Down e patogênese } \\
\text { do SARS-CoV-2. }\end{array}$ & Análise qualitativa \\
\hline Del Carmen Ortega et al., 2020 & $\begin{array}{l}\text { Lessons from individuals with } \\
\text { Down Syndrome during } \\
\text { COVID-19 }\end{array}$ & $\begin{array}{l}\text { Perspectiva de como as pessoas } \\
\text { com trissomia do } 21 \text { podem dar } \\
\text { exemplo às demais pessoas, } \\
\text { durante a pandemia da COVID- } \\
19 .\end{array}$ & Estudo observacional \\
\hline Espinosa, 2020 & $\begin{array}{l}\text { Down Syndrome and COVID- } \\
\text { 19: A Perfect Storm? }\end{array}$ & $\begin{array}{l}\text { Descreve porque os indivíduos } \\
\text { com Síndrome de Down devem } \\
\text { ser considerados uma população } \\
\text { de risco para COVID-19 grave. }\end{array}$ & Delineamento de perspectiva \\
\hline Emami, 2021 & $\begin{array}{l}\text { COVID-19 in patients with } \\
\text { Down syndrome }\end{array}$ & $\begin{array}{l}\text { Determinar se COVID-19 está } \\
\text { associado a um quadro clínico } \\
\text { diferente ou a um curso mais } \\
\text { grave da doença em pessoas } \\
\text { com Síndrome de Down (SD). }\end{array}$ & Estudo de caso-controle \\
\hline Faundes et al., 2021 & $\begin{array}{l}\text { Consensus of the Genetics } \\
\text { Branch of the Chilean Society of } \\
\text { Pediatrics on the prioritization } \\
\text { of people with Down syndrome } \\
\text { and rare diseases for vaccination } \\
\text { against SARS-CoV-2 }\end{array}$ & $\begin{array}{l}\text { Enfatizar que indivíduos com } \\
\text { Síndrome de Down e doenças } \\
\text { raras devem ser priorizados na } \\
\text { ordem de vacinação contra a } \\
\text { COVID-19. }\end{array}$ & $\begin{array}{l}\text { Consenso do Ramo de Genética } \\
\text { da Sociedade Chilena de } \\
\text { Pediatria }\end{array}$ \\
\hline Figueiredo Filho; Silva 2021 & $\begin{array}{l}\text { Social distancingand severe } \\
\text { acute respiratory syndrome } \\
\text { Coronavirus } 2 \text { transmission: A } \\
\text { case study from Araraquara, São } \\
\text { Paulo, Brazil }\end{array}$ & \begin{tabular}{llr} 
Avaliar o & impacto & do \\
distanciamento & \multicolumn{2}{c}{ social } \\
disseminação & da & doença \\
Coronavírus. & &
\end{tabular} & Análise temporal \\
\hline Kantar et al., 2020 & 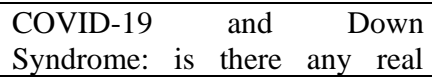 & $\begin{array}{lccc}\text { Apresentar } & \text { dois } & \text { casos } & \text { de } \\
\text { indivíduos } & \text { com Síndrome } & \text { de }\end{array}$ & de caso \\
\hline
\end{tabular}




\begin{tabular}{|c|c|c|c|}
\hline & $\begin{array}{l}\text { reason to worry? Two case } \\
\text { reports with severe course }\end{array}$ & $\begin{array}{lcc}\text { Down e } & \text { COVID-19 } & \text { que } \\
\text { evoluíram da forma grave. } & \\
\end{array}$ & \\
\hline Kim-Hellmuth et al., 2021 & $\begin{array}{l}\text { SARS-CoV-2 Triggering Severe } \\
\text { Acute Respiratory Distress } \\
\text { Syndrome and Secondary } \\
\text { Hemophagocytic } \\
\text { Lymphohistiocytosis in a 3- } \\
\text { Year-Old Child with Down } \\
\text { Syndrome }\end{array}$ & $\begin{array}{l}\text { Relatar um paciente pediátrico } \\
\text { com Síndrome de Down com } \\
\text { COVID-19 que desenvolveu } \\
\text { uma síndrome hiperinflamatória, } \\
\text { SRA grave e LHH secundária, } \\
\text { necessitando de internação na } \\
\text { UTIP e tratamento com } \\
\text { esteróides, IVIG e Remdesivir. }\end{array}$ & Relato de caso \\
\hline Krishnan et al., 2020 & $\begin{array}{l}\text { SARS-CoV-2 Infection in } \\
\text { Patients with Down Syndrome, } \\
\text { Congenital Heart Disease, and } \\
\text { Pulmonary Hypertension: is } \\
\text { Down Syndrome a Risk Factor? }\end{array}$ & \begin{tabular}{lrr} 
Descrever três & pacientes com \\
Síndrome de & \multicolumn{2}{c}{ Down } \\
insuficiência & \multicolumn{2}{c}{ respiratória } \\
secundária à & infecção por \\
Coronavírus. & & \\
\end{tabular} & Relato de caso \\
\hline Marques et al., 2021 & $\begin{array}{lccr}\text { Ações } & \text { do } & \text { Projeto } & \text { Carinho } \\
\text { durante o } & \text { isolamento social } \\
\text { ocasionado pela } & \text { COVID-19: } \\
\text { Grupo Downdança }\end{array}$ & $\begin{array}{l}\text { Descrever o efeito das } \\
\text { atividades remotas de dança nas } \\
\text { pessoas com Síndrome de } \\
\text { Down, participantes do grupo } \\
\text { Downdança do Projeto Carinho } \\
\text { ESEF/UFPel, no período de } \\
\text { isolamento social ocasionado } \\
\text { pela COVID-19. }\end{array}$ & Análise do projeto Downdança \\
\hline Newman et al., 2020 & $\begin{array}{l}\text { Trissomy } 21 \text { and Coronavirus } \\
\text { Disease } 2019 \text { in Pediatric } \\
\text { Patients }\end{array}$ & $\begin{array}{l}\text { Descrever quatro casos de } \\
\text { crianças com trissomia do } \\
\text { cromossomo } 21 \text { infectadas pelo } \\
\text { Coronavírus } 2 .\end{array}$ & Relato de caso \\
\hline Olsen, 2020 & $\begin{array}{l}\text { Downs Syndrom og COVID-19 } \\
\text { - en oversett risikogruppe? }\end{array}$ & $\begin{array}{l}\text { Explorar sobre a relação entre } \\
\text { indivíduos com Síndrome de } \\
\text { Down e Coronavírus e expor } \\
\text { medidas para prevenir a saúde } \\
\text { dessa classe. }\end{array}$ & Artigo de opinião \\
\hline Paz-Maldonato, 2021 & $\begin{array}{l}\text { Down Syndrome And COVID- } \\
\text { 19: A Brief Perspective }\end{array}$ & $\begin{array}{l}\text { Expor medidas a fim de } \\
\text { contribuir para melhoria da } \\
\text { qualidade de vida de pessoas } \\
\text { com Síndrome de Down, frente } \\
\text { ao Coronavírus. }\end{array}$ & Artigo de opinião \\
\hline Russo et al., 2020 & $\begin{array}{l}\text { Ações contra a COVID-19 na } \\
\text { população com Síndrome de } \\
\text { Down }\end{array}$ & $\begin{array}{l}\text { Apresentar como o Coronavírus } \\
\text { e o isolamento social impacta na } \\
\text { vida de indivíduos com } \\
\text { Síndrome de Down. }\end{array}$ & Carta científica \\
\hline Santoro et al., 2021 & $\begin{array}{l}\text { Pneumonia and respiratory } \\
\text { infections in Down syndrome: A } \\
\text { scoping review of the literature }\end{array}$ & $\begin{array}{l}\text { Revisar a literatura para avaliar } \\
\text { lacunas e abordar questões- } \\
\text { chave. }\end{array}$ & Revisão de literatura \\
\hline Vita et al., 2020 & $\begin{array}{l}\text { Down Syndrome patients with } \\
\text { COVID-19 pneumonia: A high- } \\
\text { risk category for unfavourable } \\
\text { outcome }\end{array}$ & $\begin{array}{l}\text { Relatar dois casos de Doença } \\
\text { por Vírus Corona-19 (COVID- } \\
\text { 19) em pacientes com Síndrome } \\
\text { de Down (SD) e descrever a } \\
\text { identificação, diagnóstico, curso } \\
\text { clínico e manejo da infecção. }\end{array}$ & Relato de caso \\
\hline
\end{tabular}

Fonte: Autores.

A partir do Quadro 1 é possível inferir que vários artigos concordam ao dizer quanto aos maiores problemas que afetam a população com Síndrome de Down e o porquê dessa população ser do grupo de risco ante a infecção pelo Coronavírus. Além disso, alguns estudos demonstram a preocupação com a qualidade de vida dessas pessoas diante do isolamento social vivenciado nos últimos tempos, já que esse grupo necessita de socialização.

Os indivíduos com SD são, de fato, prediletos como tendo maior risco de evolução para gravidade perante uma infecção por Coronavírus, visto que eles apresentam maior prevalência de comorbidades associadas ao pior prognóstico, como diabetes, cardiopatias e problemas respiratórios, apresentando maiores taxas de infecções por vias respiratórias e mortalidade por pneumonia e sepse, devido a desregulação imunológica e chuva de citocinas (De Toma; Dierssen, 2021; Olsen, 2020).

Nos primeiros anos de vida, indivíduos com SD apresentam evidências de imunodeficiência combinada, com 
diminuição dos níveis de interleucinas pró-inflamatórias (IL-2) e níveis elevados de interleucinas antiinflamatórias (IL-4, IL-6, IL-10, TNF- $\alpha$ ) associado à involução tímica acelerada, o que modifica a função imune mediada por células. Tais fatores aliados à presença de cardiopatias congênitas, anormalidades anatômicas e fisiológicas das vias aéreas, hipotonia e microaspirações explicariam a maior incidência de infecções respiratórias, principalmente na infância (Faundes et al., 2021; Kantar et al., 2020; De Toma; Dierssen, 2021).

O cromossomo 21 abriga vários genes envolvidos na orquestração das respostas imunológicas - como receptores de IFN- $\gamma$, que servem como subunidades de receptor para as interleucinas (IL) 10, 22 e 26 - e sua superexpressão induz um sistema imunológico hiperativo, sendo que muitas vezes as células imunes e as não imunes são hipersensíveis à estimulação do IFN- $\gamma$, mesmo sem sinais de infecção; o que torna esse grupo de pessoas mais suscetível a infecção pelo SARS-CoV-2, visto que eles demonstraram uma atividade crônica aumentada do IFN- $\gamma$. Outrossim, pessoas com SD possuem uma triplicação do gene TMPRSS2, localizado em Chr21q2214, que codifica uma serina protease que ativa proteoliticamente a proteína S que, por sua vez, irá interagir com a ECA II, a qual permitirá a entrada do vírus na célula hospedeira (Espinosa, 2020).

Tido isso, estudos demonstraram que essa classe tem prevalência sete vezes maior de pneumonia e apresentam gravidade aumentada em infecções respiratórias, com maior risco de hospitalização, necessidade de terapia intensiva e uso de ventilação mecânica, quando comparados ao grupo controle. Em infecções pelo SARS-CoV-2, ensaios estimaram que a incidência de hospitalização na população com SD foi 8,9 vezes maior (IC: 4,00 - 20,0) do que na população geral, para indivíduos entre 30 e 64 anos e esses pacientes eram, em média, 10 anos mais jovens do que o restante da população hospitalizada (Kantar et al., 2020; Santoro, 2020; Olsen, 2020).

Embora as informações sobre a epidemiologia geral e as características clínicas dos pacientes com SD e infecção pelo Coronavírus ainda sejam escassas, essa população deve ser considerada de alto risco. Três associações brasileiras [Fundação Síndrome de Down (FSD), Pontifícia Universidade Católica de Campinas (PUC-Campinas) e Sociedade Brasileira de Cardiologia (SBC)] produziram, diante das medidas de contenção/isolamento social, materiais de apoio para jovens com SD e suas famílias, com o objetivo de ajudar a combater ou diminuir o contágio - sabido que indivíduos com SD compartilham uma tendência a imitar e repetir comportamentos e esse padrão de função cognitiva associado ao fenótipo comportamental poderia promover a adesão das práticas preventivas ao SARS-CoV-2 (Del Carmen Ortega, 2020; Russo et al., 2020). Assim, também foi orientado aos jovens com SD que produzissem vídeos que elucidassem as práticas de prevenção contra o Coronavírus, de forma que se preservasse a interação, comunicação e socialização entre eles - fatores importantes para o desenvolvimento deles e que foram afetados com o avanço da pandemia.

Um estudo realizado pelo projeto "Downdança", revelou que de 14 estudantes que praticavam atividades de dança antes da pandemia, 10 pararam totalmente suas atividades durante os períodos de quarentena e 5 perceberam aumento de peso corporal e dores corporais, além de situações de estresse - o que demonstra como as consequências da pandemia atingem desfavoravelmente a saúde de indivíduos com SD, tanto frente ao isolamento social (que pode propiciar baixos níveis de atividade física e sedentarismo, corroborando para a tendência do desenvolvimento de obesidade, HAS e diabetes), tanto quanto às chances de agravamento perante uma contaminação pelo SARS-CoV-2 (Marques et al., 2021; Correia et al., 2020).

Portanto, mesmo sendo o isolamento social o meio mais eficaz para o combate à pandemia, é importante a manutenção das atividades físicas aeróbicas durante esse período, através do desenvolvimento de um trabalho coletivo entre familiares e cuidadores para manutenção de rotinas relacionadas à higiene e aos cuidados pessoais - obtendo-se melhorias dos hábitos alimentares e prática de esportes e atividades recreativas, que propicia a diminuição da obesidade e do estresse, além de fortificar o organismo para uma possível infecção viral - e prestação da devida atenção aos métodos de prevenção e ao tratamento oferecido, frente uma infecção por SARS-CoV-2, a fim de diminuir as possíveis complicações que a COVID-19 pode acarretar (Figueiredo Filho; Silva, 2021; Paz-Maldonato, 2021; Silva et al., 2021). Também se faz necessário e essencial 
a adesão aos meios de barreira e uso de equipamentos de proteção individual para redução da morbimortalidade dos pacientes (De Cauwer, Spaepen, 2021).

\section{Conclusão}

Indivíduos com Síndrome de Down possuem comorbidades como obesidade, diabetes tipo I, doença cardíaca congênita e alterações de imunidade - com resposta inflamatória exacerbada, principalmente frente infecções de vias respiratórias. Em patologias como a COVID-19, esses pacientes possuem forte tendência a desenvolverem a forma mais grave da doença, evoluindo para Síndrome Respiratória Aguda Grave (SARS).

Desse modo, é importante a vigilância e a capacitação dessa população e de suas famílias e cuidadores para que previnam-se de forma correta, com técnica correta de lavagem de mãos e uso de equipamentos de barreira. Embora o isolamento social seja a maneira mais eficaz de barrar a contaminação pelo vírus, é importante a manutenção e o estímulo às pessoas com SD para a continuidade da prática de exercícios físicos e interação social, mesmo que por via remota. Já que o sedentarismo e a falta de socialização também podem impactar de forma negativa na saúde desses indivíduos.

Sugere-se para estudos futuros, mais pesquisas a respeito de outras classes com síndromes genéticas, para que se possa descrever os efeitos de doenças, como a COVID-19, nessas populações.

\section{Referências}

Aquino, E. M. L., Silveira, I. H., Pescarini, J. M., Aquino, R., Souza-Filho, J. A. de, Rocha, A. dos S., Ferreira, A., Victor, A., Teixeira, C., Machado, D. B., Paixão, E., Alves, F. J. O., Pilecco, F., Menezes, G., Gabrielli, L., Leite, L., Almeida, M. da C. C. de, Ortelan, N., Fernandes, Q. H. R. F., \& Ortiz, R. J. F. (2020). Medidas de distanciamento social no controle da pandemia de COVID-19: potenciais impactos e desafios no Brasil. Ciência \& Saúde Coletiva, 25(suppl 1), 2423-2446. https://doi.org/10.1590/1413-81232020256.1.10502020

Clift, A. K., Coupland, C. A. C., Keogh, R. H., Hemingway, H., \& Hippisley-Cox, J. (2021). COVID-19 Mortality Risk in Down Syndrome: Results From a Cohort Study of 8 Million Adults. Annals of Internal Medicine, 174(4), 572-576. https://doi.org/10.7326/m20-4986

Correia, B. F., Saraiva, J. F. K., Mayeiro, M. G. de C., Soldá, M. V., Baraldi, N. R., Bustamante, T. F. O., \& Aguiar, V. B. (2020). Protagonismo de jovens com Síndrome de Down em campanha de prevenção ao SARS-CoV-2.Gestão E Desenvolvimento, (28), 301-313. https://doi.org/10.34632/gestaoedesenvolvimento.2020.9474

De Cauwer, H., \& Spaepen, A. (2020). Are patients with Down syndrome vulnerable to life-threatening COVID-19? Acta Neurologica Belgica, 121(3), 685687. https://doi.org/10.1007/s13760-020-01373-8

De Toma, I., \& Dierssen, M. (2021). Network analysis of Down syndrome and SARS-CoV-2 identifies risk and protective factors for COVID-19. Scientific Reports, 11(1). https://doi.org/10.1038/s41598-021-81451-w

Del Carmen Ortega, M., Borrel, J. M., de Jesús Bermejo, T., González-Lamuño, D., Manso, C., de la Torre, R., Mayer, M.-A., de Asúa, D. R., \& Dierssen, M. (2020). Lessons from individuals with Down syndrome during COVID-19. The Lancet Neurology, 19(12), 974-975. https://doi.org/10.1016/s14744422(20)30401-4

Emami, A., Javanmardi, F., Akbari, A., \& Asadi-Pooya, A. A. (2021). COVID-19 in patients with Down syndrome. Neurological Sciences, 42(5), 1649-1652. https://doi.org/10.1007/s10072-021-05091-8

Espinosa, J. M. (2020). Down Syndrome and COVID-19: A Perfect Storm? Cell Reports Medicine, 1(2), 100019. https://doi.org/10.1016/j.xcrm.2020.100019

Faundes, V., Pardo, R., Cammarata-Scalisi, F., Alarcon, P., Lay-Son, G., Martin, S., Faundes, V., Pardo, R., Cammarata-Scalisi, F., Alarcon, P., Lay-Son, G., \& Martin, S. (2021). Consenso de la Rama de Genética de la Sociedad Chilena de Pediatría sobre priorización de personas con Síndrome de Down y otras condiciones poco frecuentes en la Campaña de Vacunación COVID-19. Andes Pediatrica, 92(2), 309-315. https://doi.org/10.32641/andespediatr.v92i2.3716

Figueiredo Filho, D. B., \& Silva, L. E. de O. (2021). Social distancing and severe acute respiratory syndrome coronavirus 2 transmission: A case study from Araraquara, São Paulo, Brazil. Revista Da Sociedade Brasileira de Medicina Tropical, 54. https://doi.org/10.1590/0037-8682-0197-2021

Kantar, A., Mazza, A., Bonanomi, E., Odoni, M., Seminara, M., Verde, I. D., Lovati, C., Bolognini, S., \& D’Antiga, L. (2020). COVID-19 and children with Down syndrome: is there any real reason to worry? Two case reports with severe course. BMC Pediatrics, 20(1). https://doi.org/10.1186/s12887-020-02471-5

Kim-Hellmuth, S., Hermann, M., Eilenberger, J., Ley-Zaporozhan, J., Fischer, M., Hauck, F., Klein, C., Haas, N., Kappler, M., Huebner, J., Jakob, A., \& von Both, U. (2020). SARS-CoV-2 Triggering Severe Acute Respiratory Distress Syndrome and Secondary Hemophagocytic Lymphohistiocytosis in a 3-Year-Old Child With Down Syndrome. Journal of the Pediatric Infectious Diseases Society, 10(4), 543-546. https://doi.org/10.1093/jpids/piaa148

Krishnan, U. S., Krishnan, S. S., Jain, S., Chavolla-Calderon, M. B., Lewis, M., Chung, W. K., \& Rosenzweig, E. B. (2020). SARS-CoV-2 Infection in Patients with Down Syndrome, Congenital Heart Disease, and Pulmonary Hypertension: Is Down Syndrome a Risk Factor? The Journal of Pediatrics, 225, 246-248. https://doi.org/10.1016/j.jpeds.2020.06.076 
Research, Society and Development, v. 10, n. 8, e49710817355, 2021

(CC BY 4.0) | ISSN 2525-3409 | DOI: http://dx.doi.org/10.33448/rsd-v10i8.17355

Marques, A. C., Silveira, N. R., Ramson, B. P., Krüger, G. R., \& Silveira, J. R. (2020). Ações Do Projeto Carinho Durante O Isolamento Social Ocasionado Pela Covid-19: Grupo Downdança. Expressa Extensão, 26(1), 154-162. https://doi.org/10.15210/ee.v26i1.19658

Newman, A. M., Jhaveri, R., Patel, A. B., Tan, T. Q., Toia, J. M., \& Arshad, M. (2021). Trisomy 21 and Coronavirus Disease 2019 in Pediatric Patients. The Journal of Pediatrics, 228, 294-296. https://doi.org/10.1016/j.jpeds.2020.08.067

Olsen, M. B. (2020). Downs syndrom og covid-19 - en oversett risikogruppe? Tidsskrift for Den Norske Legeforening. https://doi.org/10.4045/tidsskr.20.0754

Paz-Maldonado, E. (2021). Síndrome de Down y COVID-19: Una Breve Perspectiva. Revista Ecuatoriana de Neurologia, 30(1), 15-15. https://doi.org/10.46997/revecuatneurol30100015

Russo, G. C., Bernardes, N., Baraldi, N. R., Saraiva, D. J. B., Angelis, K. D., Lantieri, C. J. B., \& Saraiva, J. F. (2020). Ações contra a Covid-19 na População com Síndrome de Down. Arquivos Brasileiros de Cardiologia, 115(5), 939-941. https://doi.org/10.36660/abc.20200685

Santoro, S. L., Chicoine, B., Jasien, J. M., Kim, J. L., Stephens, M., Bulova, P., \& Capone, G. (2020). Pneumonia and respiratory infections in Down syndrome: A scoping review of the literature. American Journal of Medical Genetics Part A, 185(1), 286-299. https://doi.org/10.1002/ajmg.a.61924

Soares, A., Dorlivete, P., Shitsuka, M., Parreira, F., \& Shitsuka, R. (2018). Metodologia Da Pesquisa Científica. https://repositorio.ufsm.br/bitstream/handle/1/15824/Lic_Computacao_Metodologia-Pesquisa-Cientifica.pdf?sequence=1

Silva, L. T., Meurer, N. C., Rodrigues, D. A. C., Rahal, Y. A., Souza, I. A. de, Caran, L. L., Cruz, I. M., Romera, L. de O., Almeida, L. B. de, Ribeiro, I. P. de A., Nunes, T. D. A., Ferracini, G. F., Polizeli, L. B., Gonçalves, F., \& Gonçalves, F. da S. (2021). Gestação e pandemia da COVID-19: Impactos no binômio materno-fetal. Research, Society and Development, 10(7), e23510716416. https://doi.org/10.33448/rsd-v10i7.16416

Vita, S., Di Bari, V., Corpolongo, A., Goletti, D., Espinosa, J., Petracca, S., Palmieri, F., Nicastri, E., Abbonizio, Agrati, C., Albarello, F., Amadei, G., Amendola, A., Antonini, M., Barbaro, R., Bartolini, B., Benigni, M., Bevilacqua, N., Bordi, L., \& Bordoni, V. (2021). Down Syndrome patients with COVID19 pneumonia: A high-risk category for unfavourable outcome. International Journal of Infectious Diseases, 103, 607-610. https://doi.org/10.1016/j.ijid.2020.11.188 